\title{
The role of visual interference in producing the long-term modality effect
}

\author{
ARTHUR M. GLENBERG, KAREN A. EBERHARDT, and TIGHE M. BELDEN \\ University of Wisconsin, Madison, Wisconsin
}

\begin{abstract}
Typically, recall of the last of a list of auditory items greatly exceeds recall of the last of a list of visual items. This modality effect has been found in serial recall, free recall, and recall using the distractor paradigm in which each to-be-remembered item is preceded and followed by distractor activity. One source of the auditory advantage may be visual interference that reduces recall of visual stimuli. In three experiments, sources of visual interference were minimized. Although this manipulation reduced the modality effect, it did not eliminate the effect.
\end{abstract}

One of the strangest findings to emerge from the memory laboratory in the last few years is the long-term modality effect (LTME). The modality effect in free recall refers to the enhanced recall of items presented auditorily, as opposed to visually, near the end of the list of items. The LTME is a similar enhancement that is found using the distractor procedure (Bjork \& Whitten, 1974). In the distractor procedure, each item in the list is preceded and followed by intervals typically filled with rehearsalpreventing distractor activity. Contrary to well-documented findings in standard free recall (e.g., Glanzer \& Cunitz, 1966), when the distractor procedure is used, the recency effect is not eliminated by the imposition of a filled retention interval between the last item and the recall test. Also, contrary to some explanations of the modality effect that propose that the effect is due to sensory (Crowder \& Morton, 1969) or short-term mechanisms (Murdock \& Walker, 1969), the LTME is not eliminated even when the retention interval is filled with overt verbal activity (Gardiner \& Gregg, 1979; Glenberg, 1984).

Recently, accounts based on retrieval processes have been proposed for the modality effect (Frankish, 1985; Frankish \& Turner, 1984), and for the LTME in particular (Gardiner, 1983; Glenberg, 1984; Glenberg \& Swanson, 1986). In all of these accounts, it is proposed that the auditory items are in some way more distinctive than the visual items, and that the retrieval system can use this distinctiveness to good advantage. For example, Glenberg and Swanson (1986) expanded Gardiner's (1983) proposal that auditory items are distinctive in the temporal domain. They proposed that time of presentation is encoded more accurately (with a finer grain or resolution) for auditory items than for visual items. To retrieve information pre-

This work was supported in part by National Science Foundation Grant BNS 8416300 to Arthur Glenberg. Experiment 1 was reported at the 1985 Meeting of the Midwestern Psychological Association in Chicago. Thanks are due Menachem Jona, who assisted in the data collection for Experiment 3. Reprints may be obtained from Arthur Glenberg, Department of Psychology, 1202 West Johnson Street, University of Wisconsin, Madison, WI 53706. sented near the end of the list, subjects construct a temporally based search set that contacts traces of items for which the encoded time of presentation is consistent with the bounds of the search set. Because time of presentation for the visual items is encoded with a coarse grain, many visual items are contacted by the end-of-list search set (including some that were not presented near the end of the list), none are distinctive, and recall is poor.

Although these retrieval accounts have had some success in accounting for the data, a simpler alternative seems to be able to explain at least the major effects of modality on memory. This alternative is a visual-interference hypothesis. In virtually all modality-effect experiments, there are many opportunities for visual information processing to exert a retroactive interfering effect on memory for the visual items. In standard free and serial recall, visual interference may arise from processing a visual recall signal, from feedback from written recall, or from incidental processing of information in the ambient visual field. In the distractor procedure, another source of visual interference is visual presentation of components of the distractor task. Thus, the modality effect may not reflect mechanisms that enhance recall of auditory items; instead, it may reflect interference with recall of visual items. The experiments reported here were designed to determine the extent to which visual interference contributes to the LTME.

In these experiments, we used a version of the distractor paradigm in which precautions were taken to eliminate sources of visual interference. First, the recall test was always oral, thereby eliminating visual interference generated during the act of recall. Previous research using the distractor procedure (Glenberg, Eberhardt, \& Petersen, 1985) demonstrated that oral recall does not adversely affect memory for auditory items. Second, the subject's visual field was restricted to a small area of a computer monitor (during both visual and auditory presentation). This precaution should eliminate the majority of visual interference that could be attributed to processing a changing ambient visual field. 


\section{EXPERIMENT 1}

Experiment 1 included two tests of the visual-interference hypothesis. The first test was provided by the very first list that subjects recalled. This list was learned under incidental learning instructions. Because the subjects were not expecting to be tested on the list, we reasoned that they would not rehearse the to-be-remembered (TBR) items, and thus there was no need for a distractor task. Consequently, the interpresentation interval (IPI) and retention interval $(\mathrm{RI})$ were unfilled, eliminating visual interference engendered by a distractor task. Unfortunately, in most of the conditions recall of the last item of the incidental list was close to $100 \%$, and thus was uninformative. For this reason we will not describe in detail the procedures used or the results obtained from this incidental list.

A second test of the visual-interference hypothesis was provided by a manipulation of the type of distractor task used subsequent to the incidental list. For half the subjects, a visually presented addition task filled the IPI and the RI. Every 2 sec the subject saw on the computer monitor a three-digit addition problem as well as an answer that was equally often the correct or incorrect sum. The subject's task was to hit one key if the sum was correct and another key if it was incorrect (see Glenberg, 1984, for details of implementation of this task). This component of the distractor task was designed to minimize rehearsal requiring capacity-demanding mechanisms. In addition, the subjects engaged in articulatory suppression by saying "blah" three to four times per second throughout the distractor intervals. This component of the distractor task was designed to minimize rehearsal by means of an articulatory loop.

The other half of the subjects engaged in an auditory tone task throughout the distractor intervals. Each tone problem lasted for $2 \mathrm{sec}$. During the first second, a series of three tones was presented through a loudspeaker located above and behind the computer monitor. Either the tone series was monotonically ascending or descending in pitch, or the pitches changed nonmonotonically. During the next second, the subject was required to press one button if the changes in pitch were monotonic and another button if the changes were nonmonotonic. As with the addition task, subjects engaged in articulatory suppression throughout the distractor interval. (Subjects articulated "blah" slightly louder than a whisper, so that the articulation could be monitored by the experimenter. These articulations did not appear to interfere with perception of the tones, which were presented at a comfortable volume.) With this tone task (and the other precautions noted before), virtually all sources of visual interference were eliminated. Thus the prediction based on the visual-interference hypothesis was that the LTME should also be eliminated.

\section{Method}

Subjects. The subjects were 48 men and women enrolled in introductory psychology courses at the University of Wisconsin-
Madison. Participation in the experiment partially fulfilled a course requirement.

Materials. The TBR stimuli consisted of 87 pairs of common, concrete, and one- and two-syllable nouns. When presented visually, a pair was displayed on the computer monitor for $3 \mathrm{sec}$. The pairs were also recorded on an Instavox RA-12 unit (ETS Systems, Champaign, IL). This computer-controlled device allows rapid (within $400 \mathrm{msec}$ ) random access to analog recordings of auditory material. Each pair was recorded within a 1,600 -msec window; however, an additional 1,400 -msec unfilled interval was appended so that the total presentation time was $3 \mathrm{sec}$. (The tones used in the tone task were also presented using the Instavox.) Pairs were randomly assigned (for each subject) to 3 three-pair practice lists, 1 six-pair incidental list, and 12 six-pair experimental lists.

Throughout the experiment, each subject's vision was restricted by a limited-vision mask. This mask consisted of a pair of rubber goggles strapped to the subject's head. The goggles were attached to a wooden rectangular tube (and a support) that was approximately $5 \times 10 \times 45 \mathrm{~cm}$ long, although the length was adjusted to suit individual subjects. This mask was attached to the computer monitor to limit the subject's vision to a $5 \times 10 \mathrm{~cm}$ area of the screen.

Procedure. Subjects participated individually. After signing a consent form, the subject listened to tape-recorded instructions characterizing the experiment as an investigation of the ability to solve word problems. Each word problem consisted of the presentation of two words. The subject was instructed to designate which of the two words referred to the larger object. For visual presentation, the subject pressed a button on the same side as the word referring to the larger object. For auditory presentation, the subject pressed the left-hand button to designate the first word as referring to the larger object and the right-hand button to designate the second word.

Subjects practiced responding on two three-pair lists. The pairs on the first list were auditory, and those on the second list were visual. Next, a six-pair list was presented (with visual TBR pairs for half the subjects), followed by an unexpected recall test. After this incidental list, half of the subjects (randomly assigned) practiced the tone distractor task and half practiced the math distractor task. Then another three-pair practice list was presented. For this list, the IPI and the RI were filled with the practiced distractor task and the subject attempted to recall the words orally. Recall was monitored by the experimenter and tape-recorded to ensure accurate scoring.

Finally, each subject studied and recalled 12 six-pair lists. Half of these lists (randomized in blocks of 4) used auditory pairs, and half used visual pairs. Before each list the subject was informed of the modality of the TBR pairs in the list. For all of these lists, the IPI preceding each TBR pair was $8 \mathrm{sec}$ (four distractor problems) and the RI following the last TBR pair was $10 \mathrm{sec}$ (five distractor problems). The RI was followed by a 45 -sec oral recall interval.

To summarize, the intentional learning part of the experiment was a 2 (type of distractor task) $\times 2$ (modality of the TBR items) $\times 6$ (serial positions) design. The first factor was manipulated between subjects, and the other factors were manipulated within subjects.

\section{Results}

We scored the number of individual words recalled, regardless of order. ${ }^{1}$ Statistical analyses were performed using a .05 probability of a Type I error. The data of major concern are presented in Figure 1.

The data from those subjects having the addition distractor task (left-hand side of Figure 1) demonstrate the salient features of the LTME: At the end of the list, recall of auditory pairs greatly exceeded recall of the visual pairs. Also, as is often the case, recall of the visual pairs exceeded recall of the auditory pairs at the beginning of the list. 


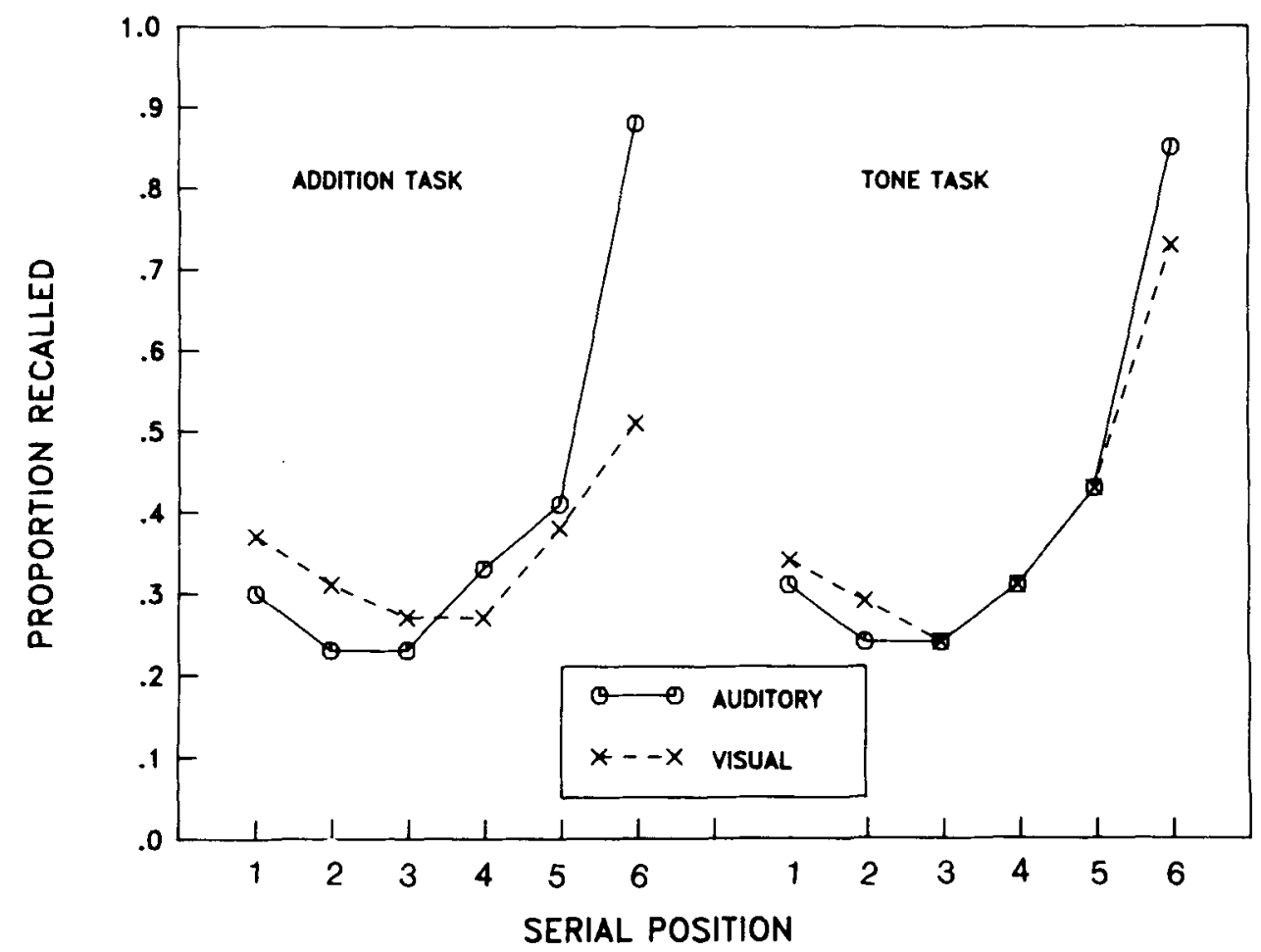

Figure 1. Experiment 1: Proportion recalled as a function of serial position, modality, and distractor task (addition or tone).

The test of the visual-interference hypothesis is provided by the data from those subjects having the tone distractor task (right-hand side of Figure 1). According to the visual-interference hypothesis, the LTME effect should be eliminated when the tone distractor task is used, because all sources of visual interference are eliminated. Contrary to this prediction, a modest LTME is still apparent.

These observations were supported by the results of statistical analyses. An analysis of all six serial positions showed a main effect of serial position $[F(5,230)=$ $102.26, M S \mathrm{e}=.032]$. Also, there were significant interactions between distractor task and modality, $[F(1,46)$ $=4.61, M S e=.018]$ and between modality and serial position $[F(5,230)=15.37, M S e=.020]$, as well as a significant three-way interaction among distractor task, modality, and serial position $[F(5,230)=3.64, M S e=$ $.020]$.

Because our main concern was with the end of the list, a second analysis was performed on the data from the last serial position. This analysis showed main effects for distractor task $[F(1,46)=8.68, M S e=.024]$ and modality $[F(1,46)=91.37, M S e=.015]$, as well as a significant interaction between these factors $[F(1,46)=22.84$, $M S \mathrm{e}=.015]$.

The clearest test of the visual-interference hypothesis is provided by a direct comparison between the level of recall of the last auditory and last visual items when the tone task was used. For this comparison, $F(1,23)=$ $13.66, M S e=.013$, demonstrating a LTME even when the tone was used and visual interference was eliminated.

\section{EXPERIMENT 2}

Interpretation of the results of Experiment 1 may be compromised by the possible ceiling effect in recall of the auditory stimuli (see Figure 1). In Experiment 2, we made use of two related findings to reduce recall. The first finding, called the ratio rule, is that the size of the increments in recall over the last few items on a list is a positive function of the ratio of the length of the IPI to the length of the RI (Bjork \& Whitten, 1974; Glenberg, Bradley, Kraus, \& Renzaglia, 1983). Decreasing the ratio will decrease the size of the recency effect. Second, the level of recall of the last item, after any RI, can be decreased by decreasing the IPI. Thus, decreasing the IPI and decreasing the ratio should reduce recall and provide a test of the visual-interference hypothesis that is free of any ceiling effects.

In Experiment 2, the IPI was reduced to $2 \mathrm{sec}$ (one distractor problem) and the RI was increased to $16 \mathrm{sec}$ (eight distractor problems). The incidental list was eliminated, and subjects were aware of the recall requirement from the outset of the experiment. As in Experiment 1, modality of the TBR items was manipulated within subjects and type of distractor task was manipulated between subjects.

\section{Method}

Subjects. Eighteen subjects were recruited from introductory psychology courses at the University of Wisconsin-Madison. Their participation partially fulfilled a course requirement. An additional 18 subjects were recruited from among the students enrolling in 
the summer session at the university. Each of these subjects was paid $\$ 3$ for participating.

Materials. A total of 78 word pairs were randomly assigned (for each subject) to 2 three-pair practice lists and 12 six-pair experimental lists. In half of the lists the TBR items were auditory and in half they were visual. Timing, the orienting task for the word pairs, and other details of presentation were the same as in Experiment 1.

Procedure. Subjects were randomly assigned to distractor task conditions, with the constraint that 9 volunteer and 9 paid subjects be assigned to each condition. After signing consent forms, subjects listened to recorded instructions. Practice was provided on the appropriate distractor task and then on a three-pair list with visual presentation of the TBR items and a three-pair list with auditory presentation of the TBR items. For these lists, as well as for the 12 experimental lists that followed, the IPI preceding each TBR item was $2 \mathrm{sec}$, the RI was $16 \mathrm{sec}$, and $45 \mathrm{sec}$ was allowed for oral recall.

\section{Results}

The results of most concern are illustrated in Figure 2. Once again, a LTME was evident when the addition task was used (left-hand panel). Data from the tone-distractor condition (right-hand panel) were used to test the visualinterference hypothesis. As in Experiment 1, the LTME was reduced in the tone-distractor condition, but it was not eliminated. Note that this reduction in the size of the LTME cannot be attributed to a ceiling effect.

Again, the statistical analyses support these conclusions. The analysis of all six positions showed a main effect of serial position $[F(5,170)=74.55, M S e=$ .029], as well as a significant interaction between modality and serial position $[F(5,170)=11.12, M S e=$
.021]. The three-way interaction among modality, serial position, and distractor conditions fell just short of statistical significance $[F(5,170)=2.13, M S e=.021, p=$ .06]. This important interaction (it indicates whether or not the size of the LTME depends on the nature of the distractor task) was statistically significant in an analysis based on the last three positions $[F(2,68)=3.72$, $M S \mathrm{e}=.020]$.

As in Experiment 1, it was important to determine if the LTME was found when the tone distractor task was used. For the 18 subjects performing the tone task in the distractor intervals, a comparison of recall of the auditory and visual items in the last serial position was significant $[F(1,17)=6.72, M S e=.024]$.

\section{EXPERIMENT 3}

One result from Experiments 1 and 2 is clear: Eliminating visual interference (as in the tone-distractor condition) does not eliminate modality differences in recall. What is not as clear is the extent to which visual interference contributes to the LTME. Judging from Figures 1 and 2, there is a sizable difference in the LTME depending on the type of task, but the causal factor cannot be uniquely indentified as visual interference because modality of the distractor task was confounded with type of distractor task (addition or pitch judgments). In this experiment, we unconfounded modality of the distractor task and type of distractor task to determine the contribution of visual interference to the LTME.

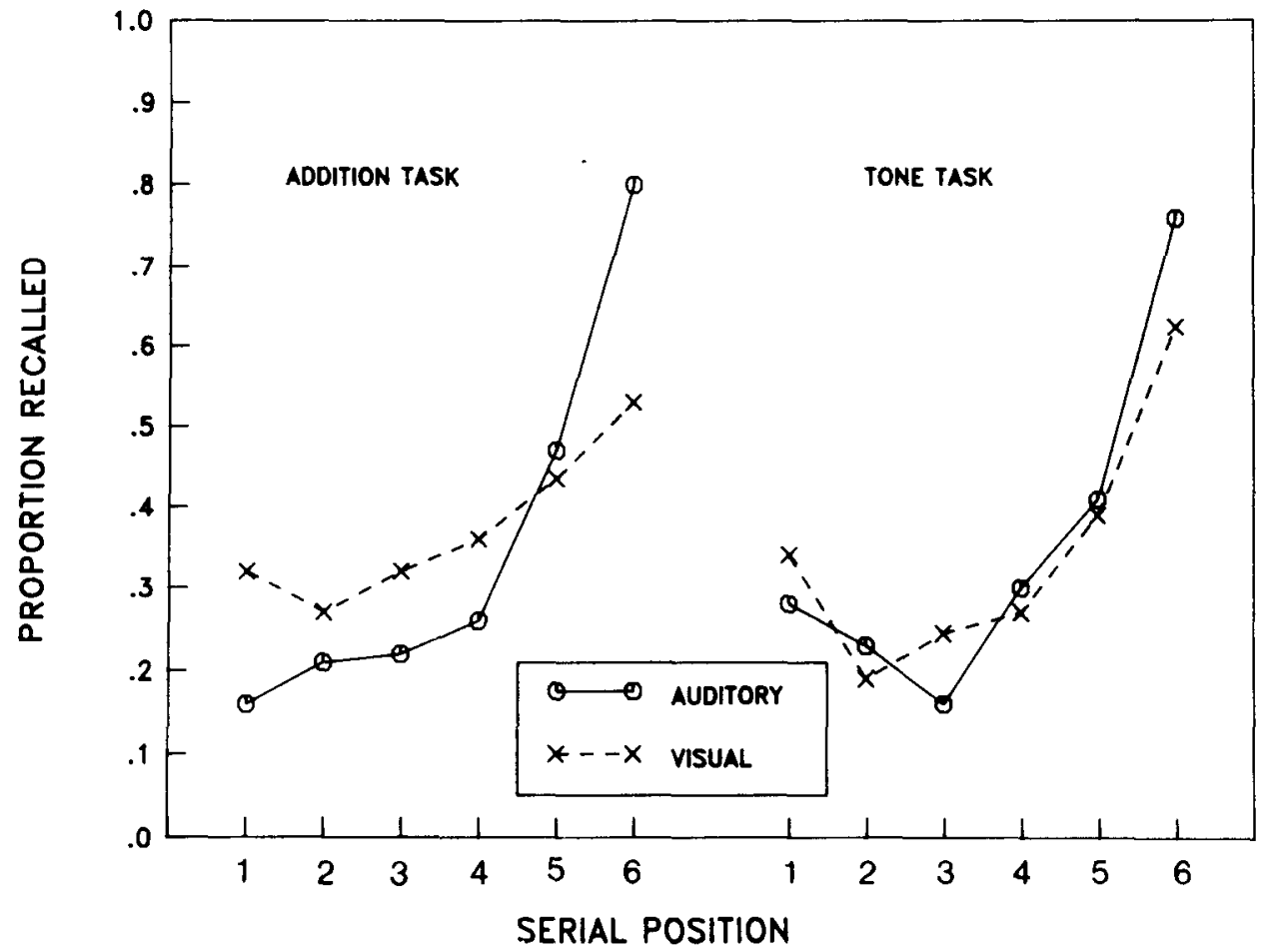

Figure 2. Experiment 2: Proportion recalled as a function of serial position, modality, and distractor task (addition or tone). 
Three distractor tasks were used. For the auditoryduration task, two tones of unequal durations were presented sequentially. The subject's task was to indicate the tone with the greater duration. This task, combined with the limited-vision mask and oral recall, should eliminate visual interference. For the visual-duration task, two lines were presented sequentially for unequal durations, and the subject was to indicate the line with the greater duration. This task introduced visual stimulation, but required the same judgment as in the auditory-duration task. For the visual-length task, two lines of unequal length were presented sequentially (for the same durations). The subject was to indicate the line with the greater spatial extent. This task introduced visual stimulation and forced the subject to attend to spatial information.

If visual interference contributes to the LTME by reducing recall of visually presented words, then the two visual tasks should produce a larger LTME than that produced by the auditory task. Furthermore, if processing of spatial information contributes to the visual interference, then the LTME should be greatest in the visual-length task.

The design of the experiment was similar to that of Experiment 2. Modality of the TBR items was manipulated within subjects and type of distractor task was manipulated between subjects. The IPI was filled with one distractor problem and the RI was filled with eight distractor problems. In this experiment, however, each distractor problem required $2.5 \mathrm{sec}$, as opposed to the $2.0 \mathrm{sec}$ used in the other experiments.

Pilot work using the three distractor tasks led us to believe that subjects were able to anticipate the end of the list and engage special rehearsal strategies. To discourage this, we manipulated (within subjects) the list length from four to six serial positions.

\section{Method}

Subjects. The 60 subjects were recruited from introductory psychology courses at the University of Wisconsin-Madison. Participation in the experiment partially fulfilled a course requirement.

Materials. A total of 68 word pairs, a subset of those used in Experiments 1 and 2, were randomly assigned (for each subject) to 2 practice lists (one with 3 pairs and one with 5 pairs) and 12 experimental lists (four at each list length of 4,5 , and 6 pairs). In half of the lists at each list length the TBR items were auditory and in half they were visual. The 12 experimental lists were randomized, with the constraint that each block of 6 lists contain one exemplar of each combination of list length and modality. The presentation modality of the pairs was indicated before each list began, but subjects were unaware of the list length. Timing, the orienting task for the word pairs, and other details of presentation were the same as in Experiments 1 and 2.

Each distractor-task problem consisted of a pair of stimuli presented sequentially for a judgment. The stimuli varied on two dimensions. One dimension was relevant to the judgment and differentiated the stimuli presented as a problem. The other dimension was irrelevant; it was the same for the pair of stimuli presented in a problem, but changed randomly between problems. The two stimuli were presented during a 1,250-msec interval and they were followed by a $1,250-\mathrm{msec}$ response interval. Subjects overtly repeated the syllable "blah" three to four times per second during the distractor problems.
For the auditory-duration problem the stimuli were two tones, and the relevant dimension was duration of the tones. Except for one constraint noted below, the duration of the shorter tone was randomly chosen from among 50 equally spaced durations in the interval (approximately) $100 \mathrm{msec}$ to (approximately) $400 \mathrm{msec}$. The duration of the other tone was determined by multiplying the duration of the first tone by a multiplier greater than 1.0. The value of this multiplier was adjusted after every five problems in an attempt to keep performance at $\mathbf{8 0 \%}$ correct. For example, if performance fell below $80 \%$ correct, the multiplier was increased to increase the difference in the durations of the tones. The one constraint on the randomly chosen duration was that it must be short enough so that the longer tone (with a duration of the randomly chosen duration times the multiplier) would have a duration of less than $400 \mathrm{msec}$. The two tones were presented in random order. Following presentation of the tones, the subject pressed a button to indicate which tone had the greater duration. For the auditory-duration problems, the irrelevant dimension was pitch. Although the two tones in any given problem had the same pitch (differing only in duration), for each problem the pitch was randomly chosen from among 40 values between approximately 150 and $1500 \mathrm{~Hz}$.

The stimuli for a visual duration problem were two lines presented for unequal amounts of time, so that the relevant dimension was again duration. Except for one constraint, the duration of the shorterduration line was chosen from the interval 100 to $400 \mathrm{msec}$ (in 1-msec increments). The duration of the other line was determined by multiplying the duration of the first line by a multiplier greater than 1.0, and this multiplier was adjusted to keep performance at $80 \%$ correct. The one constraint in choosing the shorter-duration line was that the duration of the other line be less than $400 \mathrm{msec}$. The two lines were presented in random order, and the subject pressed a button to indicate the line with the greater duration. The irrelevant dimension was length of the lines. Although the two lines in any given problem had the same length (differing only in duration), for each problem the length was randomly chosen from among 195 values, equally spaced from approximately 0.36 to $14.34 \mathrm{~cm}$.

The stimuli for the visual-length problems were identical to those for the visual-duration problems, except that the relevant and irrelevant dimensions were interchanged. Thus, in a given problem, the lengths of the lines varied (and the difference was adjusted to keep performance near $80 \%$ correct), and their durations were constant (and irrelevant). Across problems, the durations varied randomly. The subject's task was to press a button to indicate which line was longer.

Procedure. Subjects were randomly assigned to distractor-task conditions. After listening to recorded instructions, each subject practiced on the appropriate distractor task, a three-pair list with visual presentation of the TBR stimuli, and a five-pair list with auditory presentation of the TBR stimuli. For these lists, as well as for the 12 experimental lists that followed, the IPI preceding each TBR item was $2.5 \mathrm{sec}$ (one distractor problem), the RI following the last TBR item was $20 \mathrm{sec}$ (eight distractor problems), and $45 \mathrm{sec}$ was allowed for oral recall.

\section{Results}

The distractor tasks differed in modality of presentation, and they may have differed in difficulty. To rule out this potential confound, it is necessary to demonstrate that performance on the three distractor tasks was comparable. In fact, percent correct responding was 76.6, 76.5, and 74.8 in the visual-duration, visual-length, and auditoryduration tasks, respectively. These percentages were not statistically different $[F(2,57)=1.64, M S \mathrm{e}=26.05]$. Equality in the percent correct measure was achieved by 
differences in the multiplier that controlled the difference in duration or length between the two stimuli in a problem. The average multipliers were $2.49,1.49$, and 2.00 for the visual-duration, visual length, and auditory-duration tasks, respectively $[F(2,57)=3.07, M S e=1.55]$.

We scored the number of words recalled from the first serial position, the last serial position, and the two positions preceding the last. In a preliminary analysis we found that list length (four, five, or six pairs) significantly reduced recall, but failed to interact with any other variable (all interaction $F \mathrm{~s}<1.0$ ). The remaining analyses ignore list length as a factor. The data of most interest are shown in Figure 3.

The significant effects were for serial position $[F(3,171)$ $=46.70, M S e=.043 \mathrm{~J}$, indicating a large recency effect, and the modality $\times$ serial position interaction $[F(3,171)$ $=6.37, M S \mathrm{e}=.027 \mathrm{l}$, indicating a greater recency effect for auditory than for visual presentation. The effect of distractor task was not significant, nor were any of its interactions.

The statistical conclusions were similar when the analysis was confined to the last serial position. There was a modality effect $[F(1,57)=27.08, M S e=.017]$, but the main effect of distractor task and its interaction with modality of presentation were nonsignificant (both $\left.F_{\mathrm{s}}<1.0\right)$.

The conclusion to be drawn from this experiment is straightforward. When difficulty and structure of the distractor tasks are equated, there is no evidence that interference from visual characteristics of the distractor task plays a role in determining the size of the auditory advantage.

\section{GENERAL DISCUSSION}

The results of the three experiments are consistent: Visual interference cannot be the sole cause of the LTME. In all three experiments, we eliminated visual interference from the ambient stimulation (by using the limitedvision mask), from the distractor task (by using a tone distractor), and from the recall task (by using oral recall), but the LTME remained.

Experiment 3 provides the clearest demonstration of the limited role of visual interference. Adding visual (and spatial) information to the distractor task, while holding other characteristics of the task constant, had little or no effect on the size of auditory superiority at the end of the list.

In Experiments 1 and 2, the size of the modality effect varied greatly between the tone task and the addition task. Given the results of Experiment 3, it seems unlikely that visual characteristics of the distractor tasks played a large role in determining the size of the modality effect. Two factors that may have controlled the size of the modality effect are difficulty of the distractor task and meaningfulness of the stimulus features (tones vs. numbers). In fact, Glenberg et al. (1980) demonstrated that more difficult distractor tasks produced greater long-term recency than did easier tasks, and Greene (1985) suggested that grouping based on meaningful features plays a role in the LTME.

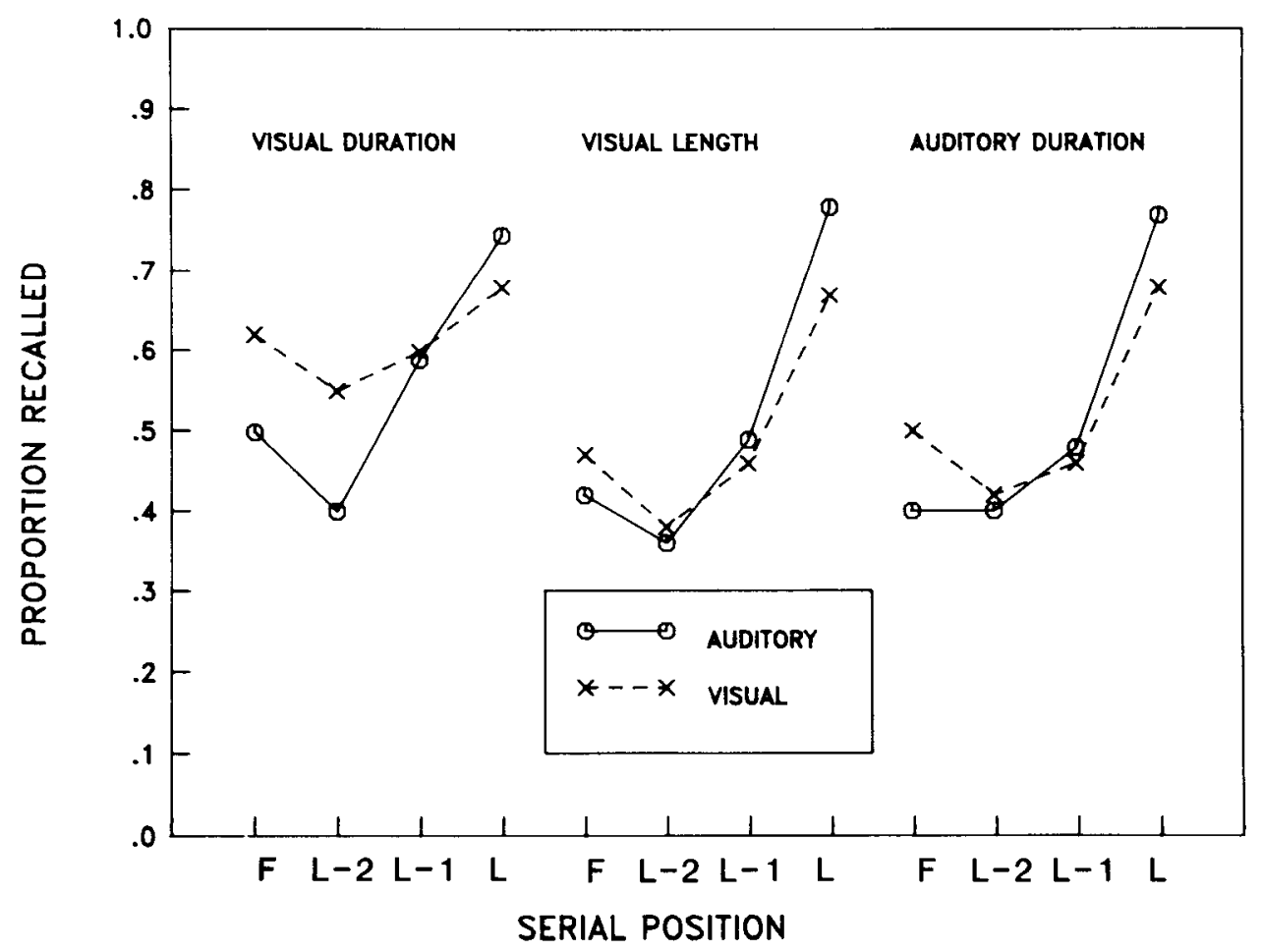

Figure 3. Experiment 3: Proportion recalled from the first (F), second to last (L-2), next to last (L-1), and last (L) serial positions. 
Nairne and McNabb (1985) suggested that the modality effect does not reflect anything special about auditory stimulation (because enhanced recency can be found in the absence of sound); instead, it reflects a type of visual inferiority. Although their general hypothesis remains secure, the results presented in this article rule out one source of visual inferiority-interference from visual characteristics of the distractor task.

\section{REFERENCES}

BJORK, R. A., \& WHITTEN, W. B. (1974). Recency-sensitive retrieval processes in long-term free recall. Cognitive Psychology, 6, 173-189.

Crowder, R. G., Morton, J. (1969). Precategorical acoustic storage (PAS). Perception \& Psychophysics, 5, 365-377.

FranKISH, C. (1985). Modality-specific grouping effects in short-term memory. Journal of Memory \& Language, 24, 200-209.

Frankish, C., \& TURNER, J. (1984). Delayed suffix effect at very short delays. Journal of Experimental Psychology: Learning, Memory, \& Cognition, 10, 767-777.

Gardiner, J. M. (1983). On recency and echoic memory. Philosophical Transactions of the Royal Society of London, 302, 267-282.

Gardiner, J. M., \& GreGG, V. H. (1979). When auditory memory is not overwritten. Journal of Verbal Learning \& Verbal Behavior, 18, 705-719.

Gardiner, J. M., GregG, V. H., \& Gardiner, M. M. (1984). Concerning some more evidence of an auditory advantage in prerecency as well as recency recall. American Journal of Psychology, 97, 593-604.

Glanzer, M., \& CunITZ, A. R. (1966). Two storage mechanisms in free recall. Journal of Verbal Learning \& Verbal Behavior, 5, 351-360.

GLENBERG, A. M. (1984). A retrieval account of the long-term modal- ity effect. Joumal of Experimental Psychology: Leaming, Memory, \& Cognition, 10, 16-31.

Glenberg, A. M., Bradley, M. M., Kraus, T. A., \& Renzaglia, G. J. (1983). Studies of the long-term recency effect: Support for a contextually guided retrieval hypothesis. Joumal of Experimental Psychology: Learning, Memory, \& Cognition, 9, 231-255.

Glenberg, A. M., Bradley, M. M., Stevenson, J. A., Kraus, T. A., TKachuK, M. J., Gretz, A. L., Fish, J. H., \& TURPIN, B. M. (1980). A two-process account of long-term serial position effects. Journal of Experimental Psychology: Human Learning \& Memory, 6, 355-369.

Glenberg, A. M., Eberhardt, K. A., \& Petersen, G. L. (1985). Differential influence of recall modality and post-list instruction modality on the long-term modality effect. American Journal of Psychology, 98, 569-578.

GlenberG, A. M., \& SWANson, N. C. (1986). A temporal distinctiveness theory of recency and modality effects. Journal of Experimental Psychology: Learning, Memory, \& Cognition, 12, 1-13.

GreENE, R. L. (1985). Constraints on the long-term modality effect. Journal of Memory \& Language, 24, 526-541.

Murdock, B. B., JR., \& W WLKER, K. D. (1969). Modality effect in free recall. Joumal of Verbal Leaming \& Verbal Behavior, 8, 665-676. NaIRNE, J. S., \& MCNABB, W. L. (1985). More modality effects in the absence of sound. Journal of Experimental Psychology: Learning, Memory, \& Cognition, 11, 596-604.

\section{NOTE}

1. Free-recall instuctions and scoring were used to be consistent with previous investigations of the LTME. Gardiner, Gregg, and Gardiner (1984) and Glenberg (1984) found that instructions for free or serial recall do not greatly affect the LTME (but see Greene, 1985).

(Manuscript received December 23, 1985; revision accepted for publication March 23, 1987.) 